\title{
Synthesis, Properties and Structures of Gallium(III) and Indium(III) Halide Complexes with Neutral Pnictine Coordination
}

Kelsey R. Cairns, Victoria K. Greenacre, Laura A. Grose, William Levason, Gillian Reid*and Fred Robinson

School of Chemistry, University of Southampton, Southampton SO17 1BJ. U.K.

(* corresponding author: G.Reid@soton.ac.uk)

Keywords: Stibine complexes: arsine complexes: gallium: indium: X-ray structures

\begin{abstract}
A series of Group 13 halide complexes of $\mathrm{Sb}^{n} \mathrm{Bu}_{3},\left[\mathrm{GaX}_{3}\left(\mathrm{Sb}^{n} \mathrm{Bu}_{3}\right)\right]$ and $\left[\ln \mathrm{X}_{3}\left(\mathrm{Sb}^{n} \mathrm{Bu}_{3}\right)\right](\mathrm{X}=\mathrm{Cl}, \mathrm{Br}, \mathrm{I})$ have been prepared by reaction of the appropriate trihalide with $\mathrm{Sb}^{n} \mathrm{Bu}_{3}$ in $n$-hexane or $\mathrm{CH}_{2} \mathrm{Cl}_{2}$ solution, and characterised by microanalysis, IR, ${ }^{1} \mathrm{H},{ }^{13} \mathrm{C}\left\{{ }^{1} \mathrm{H}\right\}$ and ${ }^{71} \mathrm{Ga} N M R$ spectroscopy. X-Ray crystal structures are reported for $\left[\ln X_{3}\left(\mathrm{Sb}^{n} \mathrm{Bu}_{3}\right)\right](\mathrm{X}=\mathrm{Cl}, \mathrm{Br})$ and $\left[\mathrm{GaX}_{3}\left(\mathrm{Sb}^{n} \mathrm{Bu}_{3}\right)\right]$. Similar pseudotetrahedral complexes of $\mathrm{AsEt}_{3},\left[\mathrm{GaCl}_{3}\left(\mathrm{AsEt}_{3}\right)\right]$, $\left[\mathrm{InCl}_{3}\left(\mathrm{AsEt}_{3}\right)\right]$ and the five-coordinate $\left[\mathrm{InCl}_{3}\left(\mathrm{AsEt}_{3}\right)_{2}\right]$ were also obtained and their structures determined. Attempts to use $\left[\mathrm{InCl}_{3}\left(\mathrm{SbR}_{3}\right)\right]$ and $\left[\mathrm{GaCl}_{3}\left(\mathrm{SbR}_{3}\right)\right]$ $\left(\mathrm{R}={ }^{\mathrm{n}} \mathrm{Bu}, \mathrm{Et}\right)$ as single source precursors for low pressure CVD growth of InSb or GaSb films were unsuccesful, instead producing elemental antimony, while $\left[\mathrm{GaCl}_{3}\left(\mathrm{AsEt}_{3}\right)\right]$ and $\left[\mathrm{InCl}_{3}\left(\mathrm{AsEt}_{3}\right)\right]$ failed to produce any deposition under similar conditions.
\end{abstract}

\section{Introduction}

After many years of relative neglect, the coordination chemistry of transition metal stibine ( $\left.\mathrm{SbR}_{3}\right)$ complexes has received much more detailed attention in the last twenty-five years, revealing significant differences to the much-studied phosphine and arsine analogues $[1,2]$. Notable features of stibines include their ability to behave as bridging ligands between two metal centres $[3,4]$ and their tendency to generate hypervalent coordination, where a stibine functioning as a $\sigma$-donor to a metal centre, simultaneously behaves as a $\sigma$-acceptor towards another donor group, including halide, amine, ether, triflate, etc. [5]. In contrast, the chemistry of stibine complexes with p-block 
centres remains little explored [6], with the mostly scattered reports in the older literature lacking detailed characterisation. Complexes of the triel elements (Group 13) include adducts of the trialkyls $\left[\mathrm{MR}_{3}\left(\mathrm{SbR}_{3}{ }^{\prime}\right)\right]\left(\mathrm{M}=\mathrm{Al}, \mathrm{Ga}, \mathrm{In} ; \mathrm{R}, \mathrm{R}^{\prime}\right.$ alkyl), which are potential precursors to thin film or nanoparticle MSb materials [6]. We recently reported a detailed examination of the triel trihalide complexes $\left[\mathrm{MX}_{3}\left(\mathrm{SbR}_{3}{ }^{\prime}\right)\right](\mathrm{X}=\mathrm{Cl}, \mathrm{Br}, \mathrm{I})$, which established that only 1:1 complexes form and that stability falls with the halide involved, $\mathrm{Cl}>\mathrm{Br}>\mathrm{I}$ [ 7].

Gallium and indium antimonide (GaSb, InSb) are III-V semiconductors of major technological significance and are attractive materials for optoelectronic devices due to their narrow band gap and high electron carrier mobility $[8,9,10]$. For uses in optoelectronic devices, thin films are required. These have been obtained by a variety of techniques including flash evaporation, sputtering, molecular beam epitaxy, electrodeposition and chemical vapour deposition $[11,12,13,14]$. Mixtures of $\mathrm{MR}_{3}$ and $\mathrm{SbR}_{3}{ }^{\prime}$ provide dual source chemical vapour deposition reagents [6], whilst rare single source precursors include the stibide trimers, $\left[\left\{\mathrm{Me}_{2} \ln \left(\mu-\mathrm{Sb}^{\mathrm{t}} \mathrm{Bu} \mathrm{u}_{2}\right)\right\}_{3}\right]$ and $\left[\left\{\mathrm{Me}_{2} \mathrm{Ga}\left(\mu-\mathrm{Sb}^{t} \mathrm{Bu}_{2}\right)\right\}_{3}\right][15]$. Stibine complexes of triel trihalides could also potentially function as single source low pressure chemical vapour deposition (LPCVD) reagents. This would have the advantage of avoiding the need for the pyrophoric triel trialkyls. As a parallel, we have previously shown that high quality films of $\mathrm{Ga}_{2} \mathrm{Se}_{3}$ and $\mathrm{Ga}_{2} \mathrm{Te}_{3}$ can be made by $\mathrm{LPCVD}$ from $\mathrm{GaCl}_{3}$ or $\operatorname{lnCl} 3$ complexes of some neutral selenoether or telluroether ligands [16]. Here we report Ga and In complexes of $\mathrm{Sb}^{n} \mathrm{Bu}_{3}$ and attempts to deposit gallium and indium stibides from complexes of $\mathrm{Sb}^{n} \mathrm{Bu}_{3}$ and $\mathrm{SbEt}_{3}$ [7]. The synthesis and structures of $\left[\mathrm{InCl}_{3}\left(\mathrm{AsEt}_{3}\right)_{n}\right](\mathrm{n}=1,2)$ and $\left[\mathrm{GaCl}_{3}\left(\mathrm{AsEt}_{3}\right)\right]$ are also reported for comparison, revealing trends in speciation as a function of the donor atom type.

\section{Experimental}

Infrared spectra were recorded as Nujol mulls between Csl plates using a PerkinElmer 100 spectrometer over the range $4000-200 \mathrm{~cm}^{-1} .{ }^{1} \mathrm{H}(400 \mathrm{Mhz}),{ }^{13} \mathrm{C}\left\{{ }^{1} \mathrm{H}\right\}(100.6 \mathrm{Mhz}),{ }^{31} \mathrm{P}\left\{{ }^{1} \mathrm{H}\right\}(161.9$ $\mathrm{MHz}),{ }^{71} \mathrm{Ga}(122.0 \mathrm{MHz})$, and ${ }^{115} \mathrm{In}$ (87.6 MHz) NMR spectra were recorded from $\mathrm{CH}_{2} \mathrm{Cl}_{2} / \mathrm{CD}_{2} \mathrm{Cl}_{2}$, solutions using a Bruker AV400 spectrometer and referenced to TMS via the residual solvent resonance, $85 \% \mathrm{H}_{3} \mathrm{PO}_{4},\left[\mathrm{Ga}\left(\mathrm{H}_{2} \mathrm{O}\right)_{6}\right]^{3+}$ and $\left[\mathrm{In}\left(\mathrm{H}_{2} \mathrm{O}\right)_{6}\right]^{3+}$ in $\mathrm{H}_{2} \mathrm{O} / \mathrm{D}_{2} \mathrm{O}$ at pH 1, respectively. Microanalyses were undertaken at London Metropolitan University. Hexane and diethyl ether were dried by distillation from sodium and $\mathrm{CH}_{2} \mathrm{Cl}_{2}$ from $\mathrm{CaH}_{2}$, and all preparations were carried out under 
rigorously anhydrous conditions via a dry dinitrogen atmosphere and standard Schlenk and glovebox techniques. $\mathrm{GaCl}_{3}, \mathrm{InCl}_{3}$, and $\mathrm{InBr}_{3}$ were purchased from Sigma-Aldrich, $\mathrm{GaBr}_{3}$ from Alfa Aesar, $\mathrm{Gal}_{3}$ from Strem, and Inl 3 from Fisher; and used as received. The complexes of $\mathrm{SbEt}_{3}$ were made as described [7].

\section{Caution! Some trialkylstibines are pyrophoric, and care should be taken to manipulate them under} strictly anaerobic conditions.

\section{$2.1 \mathrm{Sb}^{n} \mathrm{Bu}$}

Magnesium turnings $(8.5 \mathrm{~g}, 349 \mathrm{mmol})$ were stirred under nitrogen overnight. Diethyl ether ( 200 $\mathrm{mL}$ ) was added, and the stirring suspension was placed into an ice bath before a mixture of ${ }^{\mathrm{n}} \mathrm{BuBr}$ (36.3 g, $265 \mathrm{mmol}$ ) with diethyl ether $(30 \mathrm{~mL}$ ) was added dropwise. The suspension turned black and was left to stir overnight at room temperature. The mixture was cooled to $0{ }^{\circ} \mathrm{C}$ and an ethereal solution of $\mathrm{SbCl}_{3}(12.05 \mathrm{~g}, 53 \mathrm{mmol})$ was added dropwise. A grey precipitate formed, and the mixture was then refluxed for 90 mins. The mixture was cooled, hydrolysed with degassed water, and the organic layer was separated and dried with degassed magnesium sulphate before the solution was filtered and transferred to a Schlenk flask were the remaining ether was removed in vacuo, leaving a yellow oil. This was distilled at $85{ }^{\circ} \mathrm{C} / 1 \mathrm{~mm} \mathrm{Hg}$, producing a colourless oil. Yield $13.0 \mathrm{~g}, 64 \% .{ }^{1} \mathrm{H}$ $\operatorname{NMR}\left(\mathrm{CD}_{2} \mathrm{Cl}_{2}, 293 \mathrm{~K}\right): \delta=1.52\left(\mathrm{t},[2 \mathrm{H}], 3^{3} \mathrm{HH}_{H}=8 \mathrm{~Hz}, \mathrm{CH}_{2}\right), 1.45-1.33\left(\mathrm{~m},[4 \mathrm{H}], \mathrm{CH}_{2}\right), 0.91\left(\mathrm{t},[3 \mathrm{H}],{ }^{3} \mathrm{~J}_{H H}=\right.$ $\left.8 \mathrm{~Hz}, \mathrm{CH}_{3}\right) .{ }^{13} \mathrm{C}\left\{{ }^{1} \mathrm{H}\right\} \mathrm{NMR}\left(\mathrm{CD}_{2} \mathrm{Cl}_{2}, 293 \mathrm{~K}\right): \delta=30.6\left(\mathrm{CH}_{2}\right), 27.0\left(\mathrm{CH}_{2}\right), 4.8\left(\mathrm{CH}_{2}\right), 14.1\left(\mathrm{CH}_{3}\right)$.

\section{1. $\left[\mathrm{GaCl}_{3}\left(\mathrm{Sb}^{n} \mathrm{Bu} \mathrm{u}_{3}\right)\right]$}

$\mathrm{GaCl}_{3}(0.285 \mathrm{~g}, 1.60 \mathrm{mmol})$ was suspended in $\mathrm{CH}_{2} \mathrm{Cl}_{2}(10 \mathrm{~mL})$ before a solution of tributylstibine ( $0.473 \mathrm{~g}, 1.60 \mathrm{mmol})$ in $\mathrm{CH}_{2} \mathrm{Cl}_{2}(3 \mathrm{~mL})$ was added. The pale-yellow suspension was stirred for $3 \mathrm{~h}$ before the solvent was filtered off to afford a pale-yellow waxy solid, which was dried in vacuo. Yield $0.653 \mathrm{~g}, 88 \%$. Anal. Calcd for $\mathrm{C}_{12} \mathrm{H}_{27} \mathrm{Cl}_{3} \mathrm{GaSb}$ (469.2): $\mathrm{C}, 30.7 ; \mathrm{H}, 5.80$. Found: $\mathrm{C}, 30.9 ; \mathrm{H}, 5.72 \% .{ }^{1} \mathrm{H}$ $\operatorname{NMR}\left(\mathrm{CD}_{2} \mathrm{Cl}_{2}, 293 \mathrm{~K}\right): \delta=1.98\left(\mathrm{t},[2 \mathrm{H}],{ }^{3} \mathrm{~J}_{H H}=8 \mathrm{~Hz}, \mathrm{CH}_{2}\right): 1.67\left(\mathrm{~m},[2 \mathrm{H}], \mathrm{CH}_{2}\right), 1.41\left(\mathrm{~m},[2 \mathrm{H}],{ }^{3} J_{H H}=8 \mathrm{~Hz}\right.$, $\left.\mathrm{CH}_{2}\right), 0.94\left(\mathrm{t},[3 \mathrm{H}], \mathrm{CH}_{3}\right) .{ }^{13} \mathrm{C}\left\{{ }^{1} \mathrm{H}\right\} \operatorname{NMR}\left(\mathrm{CD}_{2} \mathrm{Cl}_{2}, 293 \mathrm{~K}\right): \delta=29.1\left(\mathrm{CH}_{2}\right), 26.5\left(\mathrm{CH}_{2}\right), 14.7\left(\mathrm{CH}_{2}\right), 13.7$ $\left(\mathrm{CH}_{3}\right) .{ }^{71} \mathrm{Ga} \mathrm{NMR}\left(\mathrm{CH}_{2} \mathrm{Cl}_{2} / \mathrm{CD}_{2} \mathrm{Cl}_{2}, 293 \mathrm{~K}\right): \delta=252.5$. IR (Nujol): $v=377,347 \mathrm{~cm}^{-1} \mathrm{Ga}-\mathrm{Cl}$.

\section{2. $\left[\mathrm{GaBr}_{3}\left(\mathrm{Sb}^{n} \mathrm{Bu}_{3}\right)\right]$}

$\mathrm{GaBr}_{3}(0.333 \mathrm{~g}, 1.08 \mathrm{mmol})$ was suspended in $n$-hexane $(10 \mathrm{~mL})$ before a solution of tributylstibine $(0.316 \mathrm{~g}, 1.07 \mathrm{mmol})$ in $n$-hexane $(3 \mathrm{~mL})$ was added. The pale-yellow suspension was left to stir for 
$3 \mathrm{~h}$, before the volatiles were removed under vacuum. The product then extracted with $\mathrm{CH}_{2} \mathrm{Cl}_{2}$ before drying in vacuo to afford a pale-yellow solid, which later degraded into an oil. Yield $0.460 \mathrm{~g}$, 71\%. ${ }^{1} \mathrm{H} \mathrm{NMR}\left(\mathrm{CD}_{2} \mathrm{Cl}_{2}, 293 \mathrm{~K}\right): \delta=2.12\left(\mathrm{t},[2 \mathrm{H}],{ }^{3} \mathrm{~J}_{\mathrm{HH}}=8 \mathrm{~Hz}, \mathrm{CH}_{2}\right), 1.74\left(\mathrm{~m},[2 \mathrm{H}],{ }^{3} \mathrm{~J}_{\mathrm{HH}}=8 \mathrm{~Hz}, \mathrm{CH}_{2}\right), 1.44$ $\left(\mathrm{m},[2 \mathrm{H}],{ }^{3} J_{\mathrm{HH}}=8 \mathrm{~Hz}, \mathrm{CH}_{2}\right), 0.95\left(\mathrm{t},[3 \mathrm{H}],{ }^{3} \mathrm{~J}_{\mathrm{HH}}=8 \mathrm{~Hz}, \mathrm{CH}_{3}\right) \cdot{ }^{13} \mathrm{C}\left\{{ }^{1} \mathrm{H}\right\} \mathrm{NMR}\left(\mathrm{CH}_{2} \mathrm{Cl}_{2}, 295 \mathrm{~K}\right): \delta=28.6\left(\mathrm{CH}_{2}\right)$, $26.4\left(\mathrm{CH}_{2}\right), 14.9\left(\mathrm{CH}_{2}\right), 13.7\left(\mathrm{CH}_{3}\right) .{ }^{71} \mathrm{Ga} \operatorname{NMR}\left(\mathrm{CH}_{2} \mathrm{Cl}_{2} / \mathrm{CD}_{2} \mathrm{Cl}_{2}, 293 \mathrm{~K}\right): \delta=115.9$. IR (Nujol): $v=276$, $221 \mathrm{~cm}^{-1} \mathrm{Ga}-\mathrm{Br}$.

\section{3. $\left[\mathrm{GaI}_{3}\left(\mathrm{Sb}^{n} \mathrm{Bu} u_{3}\right)\right]$}

$\mathrm{Gal}_{3}(0.428 \mathrm{~g}, 1.07 \mathrm{mmol})$ was suspended in $n$-hexane $(10 \mathrm{~mL})$ before a solution of tributylstibine $(0.315 \mathrm{~g}, 1.07 \mathrm{mmol})$ in $n$-hexane $(3 \mathrm{~mL})$ was added. The yellow suspension was left to stir for $3 \mathrm{~h}$, before the solvent was removed by filtration, to afford a pale-yellow solid which was dried in vacuo. Yield 0. 610 g, 77\%. Anal. Calcd for $\mathrm{C}_{12} \mathrm{H}_{27} \mathrm{I}_{3} \mathrm{GaSb}$ (743.5): C, 19.4; $\mathrm{H}, 3.66$. Found: C, 19.2; $\mathrm{H}, 3.74 \%$. ${ }^{1} \mathrm{H}$ NMR $\left(\mathrm{CD}_{2} \mathrm{Cl}_{2}, 293 \mathrm{~K}\right): \delta=2.11\left(\mathrm{t},[2 \mathrm{H}], \mathrm{CH}_{2}\right), 1.77\left(\mathrm{~m},[2 \mathrm{H}], \mathrm{CH}_{2}\right), 1.45\left(\mathrm{~m},[2 \mathrm{H}],{ }^{3} \mathrm{JHH}_{\mathrm{HH}}=8 \mathrm{~Hz}, \mathrm{CH}_{2}\right)$, $0.96\left(\mathrm{t},[3 \mathrm{H}], \mathrm{CH}_{3}\right) .{ }^{13} \mathrm{C}\left\{{ }^{1} \mathrm{H}\right\} \mathrm{NMR}\left(\mathrm{CD}_{2} \mathrm{Cl}_{2}, 293 \mathrm{~K}\right): \delta=28.4\left(\mathrm{CH}_{2}\right), 26.6\left(\mathrm{CH}_{2}\right), 15.2\left(\mathrm{CH}_{2}\right), 13.7\left(\mathrm{CH}_{3}\right) .{ }^{71} \mathrm{Ga}$ $\operatorname{NMR}\left(\mathrm{CH}_{2} \mathrm{Cl}_{2} / \mathrm{CD}_{2} \mathrm{Cl}_{2}, 293 \mathrm{~K}\right): \delta=236.4$. IR (Nujol): $v=230,209 \mathrm{~cm}^{-1} \mathrm{Ga}-\mathrm{I}$.

\section{4. $\left[\ln C l_{3}\left(\mathrm{Sb}^{n} \mathrm{Bu} u_{3}\right)\right]$}

$\mathrm{InCl}_{3}(0.478 \mathrm{~g}, 2.16 \mathrm{mmol})$ was dissolved in $n$-hexane $(10 \mathrm{~mL})$ before a solution of tributylstibine $(0.630 \mathrm{~g}, 2.16 \mathrm{mmol})$ in $n$-hexane $(3 \mathrm{~mL})$ was added. The white suspension was left to stir for $3 \mathrm{~h}$, before the volatiles were removed in vacuo. The product was extracted in $\mathrm{CH}_{2} \mathrm{Cl}_{2}(3 \mathrm{~mL})$ and the solvent removed in vacuo to afford a white solid. Yield $0.489 \mathrm{~g}, 44 \%$. Anal. Calcd for $\mathrm{C}_{12} \mathrm{H}_{27} \mathrm{Cl}_{3} \operatorname{lnSb}$ (514.3): C, 28.03; H, 5.29. Found: C, 28.15; H, 5.33\%. ${ }^{1} \mathrm{H}$ NMR: $\left(\mathrm{CD}_{2} \mathrm{Cl}_{2}, 293 \mathrm{~K}\right): \delta=2.20\left(\mathrm{t},[2 \mathrm{H}],{ }^{3} \mathrm{~J}_{H H}\right.$ $\left.=8 \mathrm{~Hz}, \mathrm{CH}_{2}\right), 1.75\left(\mathrm{~m},[2 \mathrm{H}],{ }^{3} \mathrm{~J}_{H H}=8 \mathrm{~Hz}, \mathrm{CH}_{2}\right), 1.44\left(\mathrm{~m},[2 \mathrm{H}],{ }^{3} \mathrm{~J}_{H H}=8 \mathrm{~Hz}, \mathrm{CH}_{2}\right), 0.96\left(\mathrm{t},[3 \mathrm{H}], 3^{3} \mathrm{JHH}_{H}=8 \mathrm{~Hz}\right.$, $\left.\mathrm{CH}_{3}\right) .{ }^{13} \mathrm{C}\left\{{ }^{1} \mathrm{H}\right\}$ NMR $\left(\mathrm{CD}_{2} \mathrm{Cl}_{2}, 295 \mathrm{~K}\right): \delta=29.2\left(\mathrm{CH}_{2}\right), 26.5\left(\mathrm{CH}_{2}\right), 15.6\left(\mathrm{CH}_{2}\right), 13.7\left(\mathrm{CH}_{3}\right) .{ }^{115} \mathrm{In} \mathrm{NMR}$ $\left(\mathrm{CH}_{2} \mathrm{Cl}_{2} / \mathrm{CD}_{2} \mathrm{Cl}_{2}, 293 \mathrm{~K}\right)$ : Not observed. IR (Nujol): $v=336,310, \mathrm{~cm}^{-1} \mathrm{In}-\mathrm{Cl}$.

\section{5. $\left[\operatorname{lnBr}_{3}\left(\mathrm{Sb}^{n} \mathrm{Bu} u_{3}\right)\right]$}

$\operatorname{lnBr}_{3}(0.381 \mathrm{~g}, 1.07 \mathrm{mmol})$ was dissolved in $n$-hexane $(10 \mathrm{~mL})$ before a solution of tributylstibine $(0.315 \mathrm{~g}, 1.07 \mathrm{mmol})$ in $n$-hexane $(3 \mathrm{~mL})$ was added. The white suspension was left to stir for $3 \mathrm{~h}$, before the solid was filtered off to afford an off-white solid which was dried in vacuo. Yield $0.524 \mathrm{~g}$, 76\%. Anal. Calcd for $\mathrm{C}_{12} \mathrm{H}_{27} \mathrm{Br}_{3} \operatorname{InSb}$ (647.6): C, 22.25; H, 4.40. Found: C, 22.09; H, 4.07\%. ${ }^{1} \mathrm{H}$ NMR $\left(\mathrm{CD}_{2} \mathrm{Cl}_{2}, 293 \mathrm{~K}\right): \delta=2.19\left(\mathrm{t},[2 \mathrm{H}],{ }^{3} \mathrm{~J}_{H H}=8 \mathrm{~Hz}, \mathrm{CH}_{2}\right), 1.75\left(\mathrm{~m},[2 \mathrm{H}],{ }^{3} J_{H H}=8 \mathrm{~Hz}, \mathrm{CH}_{2}\right), 1.44\left(\mathrm{~m},[2 \mathrm{H}],{ }^{3} \mathrm{~J}_{H H}\right.$ 
$\left.=8 \mathrm{~Hz}, \mathrm{CH}_{2}\right), 0.96\left(\mathrm{t},[3 \mathrm{H}],{ }^{3} \mathrm{~J}_{\mathrm{HH}}=8 \mathrm{~Hz}, \mathrm{CH}_{3}\right) \cdot{ }^{13} \mathrm{C}\left\{{ }^{1} \mathrm{H}\right\} \mathrm{NMR}\left(\mathrm{CD}_{2} \mathrm{Cl}_{2}, 293 \mathrm{~K}\right): \delta=29.1\left(\mathrm{CH}_{2}\right), 26.5\left(\mathrm{CH}_{2}\right)$, $15.7\left(\mathrm{CH}_{2}\right), 13.7\left(\mathrm{CH}_{3}\right) .{ }^{115}$ In NMR $\left(\mathrm{CH}_{2} \mathrm{Cl}_{2} / \mathrm{CD}_{2} \mathrm{Cl}_{2}, 293 \mathrm{~K}\right): \delta=278.2$ (broad). IR (Nujol): $v=214,211$ $\mathrm{cm}^{-1} \mathrm{In}-\mathrm{Br}$.

\section{6. $\left[\ln I_{3}\left(\mathrm{Sb}^{n} B u_{3}\right)\right]$}

$\operatorname{lnl}_{3}(0.530 \mathrm{~g}, 1.07 \mathrm{mmol})$ was suspended in $n$-hexane $(10 \mathrm{~mL})$ before a solution of tributylstibine $(0.315 \mathrm{~g}, 1.07 \mathrm{mmol})$ in $n$-hexane $(3 \mathrm{~mL})$ was added. The pale-yellow suspension was left to stir for $3 \mathrm{~h}$, before the mixture was filtered to afford a pale-yellow solid which was dried in vacuo. Yield 0.670 g, 79\%. Anal. Calcd for $\mathrm{C}_{12} \mathrm{H}_{27} \mathrm{l} 3 \mathrm{InSb}$ (788.6): C, 18.26; H, 3.45. Found: C, 18.09; H, 3.52\%. ${ }^{1} \mathrm{H}$ $\operatorname{NMR}\left(\mathrm{CD}_{2} \mathrm{Cl}_{2}, 293 \mathrm{~K}\right): \delta=2.26$ (triplet, $\left.[2 \mathrm{H}], \mathrm{CH}_{2}\right), 1.78\left(\mathrm{~m},[2 \mathrm{H}], \mathrm{CH}_{2}\right), 1.45\left(\mathrm{~m},[2 \mathrm{H}],{ }^{3} \mathrm{JHH}_{H}=4 \mathrm{~Hz}, \mathrm{CH}_{2}\right)$, $0.97\left(\mathrm{t},[3 \mathrm{H}],{ }^{3} \mathrm{HHH}_{1}=8 \mathrm{~Hz}, \mathrm{CH}_{3}\right) \cdot{ }^{13} \mathrm{C}\left\{{ }^{1} \mathrm{H}\right\} \operatorname{NMR}\left(\mathrm{CD}_{2} \mathrm{Cl}_{2}, 293 \mathrm{~K}\right): \delta=28.2\left(\mathrm{CH}_{2}\right), 26.5\left(\mathrm{CH}_{2}\right), 17.5\left(\mathrm{CH}_{2}\right)$, $13.8\left(\mathrm{CH}_{3}\right) .{ }^{115}$ In NMR $\left(\mathrm{CH}_{2} \mathrm{Cl}_{2} / \mathrm{CD}_{2} \mathrm{Cl}_{2}, 293 \mathrm{~K}\right): \delta=-235$ (broad).

\section{7. $\left[\operatorname{InCl}_{3}\left(\mathrm{PEt}_{3}\right)_{2}\right]$}

$\mathrm{InCl}_{3}$ (0.055 g, $0.250 \mathrm{mmol}$ ) was suspended in $\mathrm{CH}_{2} \mathrm{Cl}_{2}(15 \mathrm{~mL})$ before dropwise addition of $\mathrm{PEt}_{3}(0.060$ $\mathrm{g}, 0.500 \mathrm{mmol}$ ) and the mixture stirred for $3 \mathrm{~h}$. The solvent was removed, leaving a white solid which was washed with $\mathrm{n}$-hexane $(2 \times 5 \mathrm{~mL})$ before being separated and dried in vacuo. Yield $0.086 \mathrm{~g}$, 74.8\%. Anal. Required for $\mathrm{C}_{12} \mathrm{H}_{30} \mathrm{Cl}_{3} \ln \mathrm{P}_{2}$ (457.49): C, 31.35; $\mathrm{H}, 6.73$. Found: $\mathrm{C}, 31.50 ; \mathrm{H}, 6.61 \%$. ${ }^{1} \mathrm{H}$ NMR: $\left(\mathrm{CD}_{2} \mathrm{Cl}_{2}, 295 \mathrm{~K}\right) \delta=1.94\left(\mathrm{~m},[2 \mathrm{H}], \mathrm{CH}_{2}\right), 1.23\left(\mathrm{dt},[3 \mathrm{H}],{ }^{3} \mathrm{~J}_{\mathrm{HH}}=7.8 \mathrm{~Hz}, \mathrm{CH}_{3}\right) .{ }^{13} \mathrm{C}\left\{{ }^{1} \mathrm{H}\right\} \mathrm{NMR}:\left(\mathrm{CD}_{2} \mathrm{Cl}_{2}\right.$ $295 \mathrm{~K}): \delta=14.4\left(\mathrm{~d},{ }^{1} \mathrm{JPC}=18.3 \mathrm{~Hz}, \mathrm{CH}_{2}\right), 8.2\left(\mathrm{CH}_{3}\right) .{ }^{31} \mathrm{P}\left\{{ }^{1} \mathrm{H}\right\} \mathrm{NMR}:\left(\mathrm{CD}_{2} \mathrm{Cl}_{2} 295 \mathrm{~K}\right): \delta=3.5$ (br). IR (Nujol): $v=284(\mathrm{br}) \mathrm{cm}^{-1} \mathrm{In}-\mathrm{Cl}$.

\section{8. $\left[\operatorname{InCl}_{3}\left(\mathrm{AsEt}_{3}\right)\right]$}

$\mathrm{InCl}_{3}$ (0.230 g, $\left.1.04 \mathrm{mmol}\right)$ was suspended in $\mathrm{CH}_{2} \mathrm{Cl}_{2}(15 \mathrm{~mL})$ before dropwise addition of $\mathrm{AsEt}_{3}$ (0.169 g, $1.04 \mathrm{mmol}$ ) and the solution stirred for $3 \mathrm{~h}$, before the solvent was removed to leave a white solid, which was washed with $\mathrm{n}$-hexane $(2 \times 5 \mathrm{~mL})$ before being dried in vacuo. Yield $0.362 \mathrm{~g}$, 90\%. Anal. Required for $\mathrm{C}_{6} \mathrm{H}_{15} \mathrm{AsCl}_{3} \ln$ (383.28): $\mathrm{C}, 18.89 ; \mathrm{H}, 4.04$. Found: $\mathrm{C}, 18.80 ; \mathrm{H}, 3.94 \% .{ }^{1} \mathrm{H} \mathrm{NMR}\left(\mathrm{CD}_{2} \mathrm{Cl}_{2}\right.$, $295 \mathrm{~K}$ ): $\delta=2.23$ (quartet, $\left.[2 \mathrm{H}], \mathrm{CH}_{2}\right), 1.40\left(\mathrm{t},[3 \mathrm{H}],{ }^{3} \mathrm{~J}_{\mathrm{HH}}=7.7 \mathrm{~Hz}, \mathrm{CH}_{3}\right) .{ }^{13} \mathrm{C}\left\{{ }^{1} \mathrm{H}\right\} \mathrm{NMR}\left(\mathrm{CD}_{2} \mathrm{Cl}_{2}, 295 \mathrm{~K}\right): \delta$ $=15.7\left(\mathrm{CH}_{2}\right), 9.9\left(\mathrm{CH}_{3}\right)$. IR (Nujol): $v=323,313, \mathrm{~cm}^{-1} \mathrm{In}-\mathrm{Cl}$.

\section{9. $\left[\operatorname{InCl}_{3}\left(\mathrm{AsEt}_{3}\right)_{2}\right]$}

$\mathrm{InCl}_{3}$ (0.115 g, $0.52 \mathrm{mmol}$ ) was suspended in $\mathrm{CH}_{2} \mathrm{Cl}_{2}(15 \mathrm{~mL})$ before dropwise addition of AsEt $_{3}(0.170$ $\mathrm{g}, 1.04 \mathrm{mmol}$ ) and the mixture stirred for $3 \mathrm{~h}$, before the solvent was removed to leave a white solid, 
which was washed with $\mathrm{n}$-hexane $(2 \times 5 \mathrm{~mL})$ and dried in vacuo. Yield $0.207 \mathrm{~g}, 72 \%$. Anal. Required for $\mathrm{C}_{12} \mathrm{H}_{30} \mathrm{As}_{2} \mathrm{Cl}_{3} \ln$ (383.28): C, 26.39; $\mathrm{H}, 5.66$. Found: $\mathrm{C}, 26.43 ; \mathrm{H}, 5.54 \% .{ }^{1} \mathrm{H} \mathrm{NMR}\left(\mathrm{CD}_{2} \mathrm{Cl}_{2}, 295 \mathrm{~K}\right): \delta$ $=1.91$ (quartet, $\left.[2 \mathrm{H}],{ }^{3} \mathrm{~J}_{\mathrm{HH}}=7.8 \mathrm{~Hz}, \mathrm{CH}_{2}\right), 1.29\left(\mathrm{t},[3 \mathrm{H}],{ }^{3} \mathrm{~J}_{\mathrm{HH}}=7.8 \mathrm{~Hz}, \mathrm{CH}_{3}\right) .{ }^{13} \mathrm{C}\left\{{ }^{1} \mathrm{H}\right\} \mathrm{NMR}\left(\mathrm{CD}_{2} \mathrm{Cl}_{2}, 295\right.$ K): $\delta=15.3\left(\mathrm{CH}_{2}\right), 9.9\left(\mathrm{CH}_{3}\right)$. IR (Nujol): $v=332,298 \mathrm{~cm}^{-1} \mathrm{In}-\mathrm{Cl}$.

\subsection{0. $\left[\mathrm{GaCl}_{3}\left(\mathrm{AsEt}_{3}\right)\right]$}

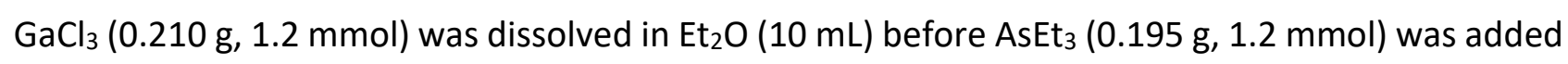
and the solution stirred for $3 \mathrm{~h}$, before removing the solvent to leave a white solid, which was dried in vacuo. Yield $0.339 \mathrm{~g}$, 84\%. Anal. Calcd for $\mathrm{C}_{6} \mathrm{H}_{15} \mathrm{AsCl}_{3} \mathrm{Ga}$ (338.2): C, 21.31; $\mathrm{H}, 4.47$. Found: C, 21.18; $\mathrm{H}, 4.32 \%$. ${ }^{1} \mathrm{H}$ NMR: $\left(\mathrm{CD}_{2} \mathrm{Cl}_{2}, 295 \mathrm{~K}\right): \delta=2.16$ (quartet, $\left.[2 \mathrm{H}],{ }^{3} \mathrm{~J}_{\mathrm{HH}}=7.7 \mathrm{~Hz}, \mathrm{CH}_{2}\right), 1.40\left(\mathrm{t},[3 \mathrm{H}],{ }^{3} \mathrm{~J}_{\mathrm{HH}}=\right.$ $\left.7.7 \mathrm{~Hz}, \mathrm{CH}_{3}\right) .{ }^{13} \mathrm{C}\left\{{ }^{1} \mathrm{H}\right\} \mathrm{NMR}\left(\mathrm{CD}_{2} \mathrm{Cl}_{2}, 295 \mathrm{~K}\right): \delta=14.2\left(\mathrm{CH}_{2}\right), 9.3\left(\mathrm{CH}_{3}\right) .{ }^{71} \mathrm{Ga} \mathrm{NMR}\left(\mathrm{CD}_{2} \mathrm{Cl}_{2}, 295 \mathrm{~K}\right): \delta=$ 265. IR (Nujol): $v=379,352 \mathrm{~cm}^{-1} \mathrm{Ga}-\mathrm{Cl}$.

\subsection{X-ray Experimental}

Data collections used a Rigaku AFC12 goniometer equipped with an enhanced sensitivity (HG) Saturn724+ detector mounted at the window of an FR-E+ SuperBright molybdenum $(\lambda=0.71073 \AA)$ rotating anode generator with VHF Varimax optics (70 micron focus) with the crystal held at $100 \mathrm{~K}$ $\left(\mathrm{N}_{2}\right.$ cryostream). Crystallographic parameters are in Table S1. Structure solution and refinement were performed using SHELX(S/L)97, SHELX-2014/7 [17] and were straightforward. CCDC reference numbers in cif format are $\left[\operatorname{lnCl}_{3}\left(\mathrm{Sb}^{n} \mathrm{Bu}_{3}\right)\right]$ 1969008, [ $\left.\operatorname{lnBr}_{3}\left(\mathrm{Sb}^{n} \mathrm{Bu}_{3}\right)\right]$ 1969009, $\left[\mathrm{GaCl}_{3}\left(\mathrm{AsEt}_{3}\right)\right]$ 1969010, $\left[\operatorname{InCl}_{3}\left(\mathrm{AsEt}_{3}\right)\right]$ 1969011, $\left[\operatorname{InCl}_{3}\left(\mathrm{AsEt}_{3}\right)_{2}\right]$ 1969012. These data can be obtained free of charge via http://www.ccdc.cam.ac.uk/conts/retrieving.html, or from the Cambridge Crystallographic Data Centre, 12 Union Road, Cambridge CB2 1EZ, UK; fax: (+44) 1223-336-033; or e-mail: deposit@ccdc.cam.ac.uk.

\subsection{Low Pressure CVD Experiments}

The apparatus used has been described elsewhere $[16,18,19]$. The precursor complex ( 20-100 mg) was loaded into the closed end of a silica tube in an $\mathrm{N}_{2}$ purged glove box. Then the silica substrates $\left(\sim 1 \times 8 \times 20 \mathrm{~mm}^{3}\right)$ were loaded in the tube, placed end-to-end. The tube was set in a furnace so that the substrates were in the heated zone and the precursor was ca. $2 \mathrm{~cm}$ away from the start of the heated zone. The tube was evacuated to $0.05 \mathrm{mmHg}$, and the furnace was heated to the requisite temperature, between 450 and $650^{\circ} \mathrm{C}$. The tube was then moved into the furnace until evaporation 
was observed and maintained until no further evaporation could be seen. It was then cooled to room temperature and the tiles were unloaded in the glove box.

X-ray diffraction (XRD) patterns were collected using a Rigaku SmartLab diffractometer $\left(\mathrm{Cu}-\mathrm{K}_{\alpha}, \lambda=\right.$ $1.5418 \AA ̊$ ) with parallel X-ray beam and a DTex Ultra 250 1D detector. Scanning electron microscopy (SEM) was performed a Philips XL30 ESEM and with an acceleration voltage of $10 \mathrm{kV}$.

\section{Results and Discussion}

3.1 Synthesis, spectroscopy and structures. The reaction of $\mathrm{Sb}^{n} \mathrm{Bu}_{3}$ with $\mathrm{GaX}_{3}$ or $\ln \mathrm{X}_{3}(\mathrm{X}=\mathrm{Cl}, \mathrm{Br}$ or I) in a 1:1 molar ratio in anhydrous $\mathrm{CH}_{2} \mathrm{Cl}_{2}$ or $n$-hexane afforded good yields of the $\left[\mathrm{GaX}_{3}\left(\mathrm{Sb}^{n} \mathrm{Bu}_{3}\right)\right]$ and [ $\left.\ln \mathrm{X}_{3}\left(\mathrm{Sb}^{n} \mathrm{Bu}_{3}\right)\right]$ as white $(\mathrm{X}=\mathrm{Cl}$ or $\mathrm{Br})$ or pale-yellow solids. Using a 1:2 ratio of $\ln \mathrm{X}_{3}: \mathrm{Sb}^{n} \mathrm{Bu}_{3}$ also produced only the $\left[\ln \mathrm{X}_{3}\left(\mathrm{Sb}^{n} \mathrm{Bu}_{3}\right)\right]$ complexes (Scheme 1), as found with other trialkylstibines [7].
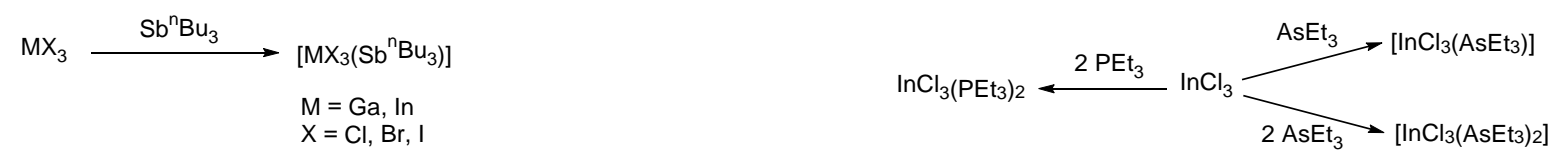

Scheme 1 Synthesis of the new pnictine complexes.

In contrast, 1:2 complexes are formed by phosphines [7,20,21,22] or arsines [23]. The spectroscopic properties of the stibine complexes closely resemble those of complexes with $\mathrm{SbEt}_{3}$ or $\mathrm{Sb}^{\mathrm{i}} \mathrm{Pr}_{3}$ [7]. Xray structures were determined for $\left[\operatorname{lnCl}_{3}\left(\mathrm{Sb}^{n} \mathrm{Bu}_{3}\right)\right]$ and $\left[\operatorname{lnBr}_{3}\left(\mathrm{Sb}^{n} \mathrm{Bu}_{3}\right)\right]$ (Figure 1) and show the expected pseudo-tetrahedral geometry at both antimony and indium. Both molecules have an eclipsed structure. The structure of the latter is the first of an $\mathrm{InBr}_{3}$-stibine complex; all crystals of the $\mathrm{InBr}_{3}$ complexes of $\mathrm{SbEt}_{3}$ or $\mathrm{Sb}^{\mathrm{i}} \mathrm{Pr}_{3}$ examined previously proved to be twinned [7]. The $\mathrm{d}(\mathrm{In}-\mathrm{Sb})$ lie in the order $\mathrm{Cl} \leq \mathrm{Br}<\mathrm{l}$, showing the expected decrease in Lewis acidity down the halide series. 


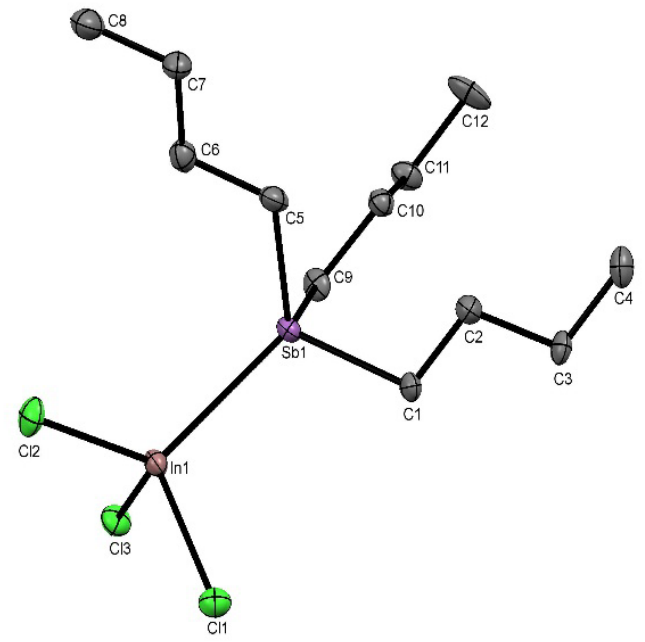

(a)

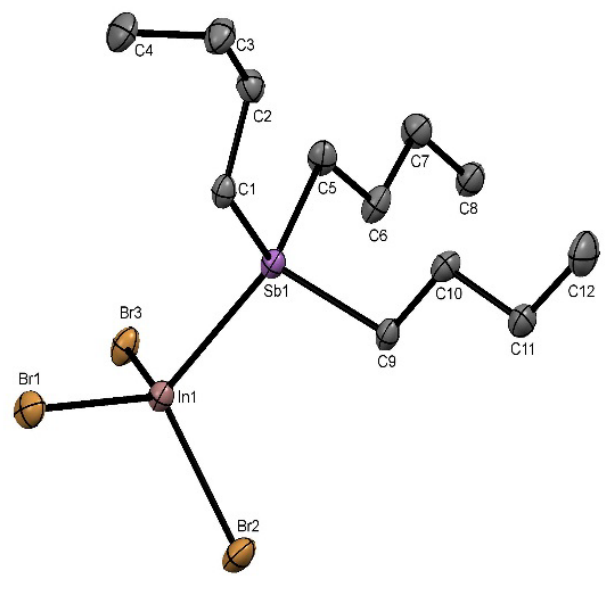

(b)

Figure 1. View of the structures of $\left[\operatorname{lnCl}_{3}\left(\mathrm{Sb}^{n} \mathrm{Bu}_{3}\right)\right](\mathrm{a})$ and $\left[\operatorname{lnBr}_{3}\left(\mathrm{Sb}^{n} \mathrm{Bu}_{3}\right)\right]$ (b) with atom numbering schemes. $\mathrm{H}$ atoms omitted for clarity and ellipsoids are shown at the $50 \%$ probability level. Selected bond lengths $(\AA)$ and angles $\left({ }^{\circ}\right)$ for $(\mathrm{a}): \mathrm{Sb} 1-\operatorname{In} 1=2.7709(7), \ln 1-\mathrm{Cl} 1=2.3900(18), \ln 1-\mathrm{Cl} 3=2.3762(17), \ln 1-\mathrm{Cl} 2=2.354(2)$, $\mathrm{Sb} 1-\mathrm{C} 1=2.137(7), \mathrm{Sb} 1-\mathrm{C} 9=2.140(7), \mathrm{Sb} 1-\mathrm{C} 5=2.127(7), \mathrm{C} 1-\mathrm{Sb} 1-\mathrm{C} 9=101.9(3), \mathrm{C} 5-\mathrm{Sb} 1-\mathrm{C} 1=104.8(3)$, $\mathrm{C} 5-\mathrm{Sb} 1-\mathrm{C} 9=105.5(3) ;(\mathrm{b}): \mathrm{Sb} 1-\ln 1=2.7791(5), \ln 1-\mathrm{Br} 3=2.5047(5), \ln 1-\mathrm{Br} 1=2.4922(5), \ln 1-\mathrm{Br} 2=$ 2.5030(6), $\mathrm{Sb} 1-\mathrm{C} 1=2.136(5), \mathrm{Sb} 1-\mathrm{C} 9=2.135(4), \mathrm{Sb} 1-\mathrm{C} 5=2.125(4), \mathrm{C} 9-\mathrm{Sb} 1-\mathrm{C} 1=104.70(18), \mathrm{C} 5-\mathrm{Sb} 1-\mathrm{C} 1=$ 108.7(2), C5-Sb1-C9 = 102.38(18).

$\operatorname{In}\left[\mathrm{InCl}_{3}\left(\mathrm{SbR}_{3}\right)\right]\left(\mathrm{R}=\mathrm{Et},{ }^{\mathrm{i}} \mathrm{Pr}\right)$ the structures revealed weak, hypervalent $\mathrm{Sb} \cdots \mathrm{Cl}$ interactions between neighbouring molecules [7]. However, in $\left[\ln \mathrm{X}_{3}\left(\mathrm{Sb}^{n} \mathrm{Bu}_{3}\right)\right]$ the nearest intermolecular $\ln \cdots \mathrm{Cl}$ or $\mathrm{Sb} \cdots \mathrm{Cl}$ distances are long, approximating to the sum of the Van der Waals radii [24]. Hypervalent interactions are typically weak [5] and have to compete with crystal packing effects, and it may be that in the latter, the larger ${ }^{\mathrm{n}} \mathrm{Bu}$ groups disfavour any intermolecular $\mathrm{Sb} \cdots \mathrm{Cl}$ interactions.

Trialkylarsine complexes of gallium(III) and indium(III) have been little studied, apart from $\left[\mathrm{GaX}_{3}\left(\mathrm{AsMe}_{3}\right)\right]$ [25], and hence we prepared $\left[\mathrm{GaCl}_{3}\left(\mathrm{AsEt}_{3}\right)\right],\left[\operatorname{InCl}_{3}\left(\mathrm{AsEt}_{3}\right)\right]$ and $\left[\operatorname{InCl}_{3}\left(\mathrm{AsEt}_{3}\right)_{2}\right]$ for comparison with the stibine complexes and as possible CVD reagents. $\left[\operatorname{lnCl}_{3}\left(\mathrm{PEt}_{3}\right)_{2}\right]$ was also prepared for comparison purposes. The spectroscopic properties and structure (Figure 2) of $\left[\mathrm{GaCl}_{3}\left(\mathrm{AsEt}_{3}\right)\right]$ closely resemble those of $\left[\mathrm{GaX}_{3}\left(\mathrm{AsMe}_{3}\right)\right][25]$. 


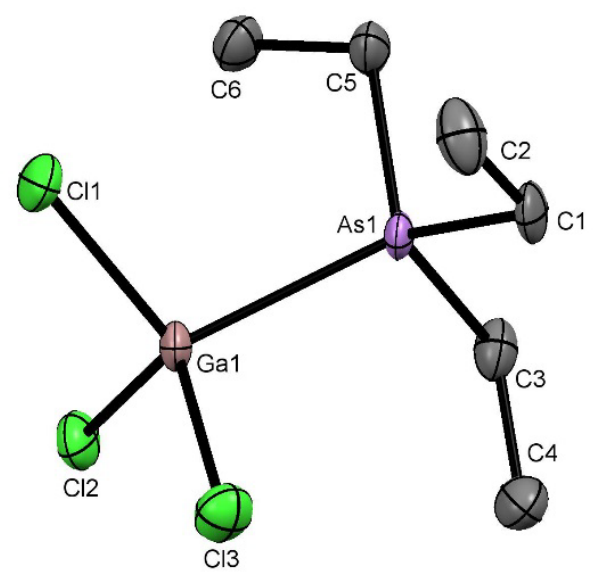

Figure 2. View of the structure of $\left[\mathrm{GaCl}_{3}\left(\mathrm{AsEt}_{3}\right)\right]$ with atom numbering scheme. $\mathrm{H}$ atoms are omitted for clarity and ellipsoids are shown at the 50\% probability level. Selected bond lengths $(\AA)$ and angles $\left({ }^{\circ}\right)$ : As1-Ga1 = 2.4279(10), Ga1-Cl1 = 2.1685(18), Ga1-Cl2 = 2.1813(19), Ga1-Cl3 = 2.169(2), C1-As1-C3 = 107.4(3), C5-As1-C1 = 106.9(3), C5-As1-C3 = 106.9(3).

The structures of the four-coordinate $\left[\mathrm{InCl}_{3}\left(\mathrm{AsEt}_{3}\right)\right]$ (pseudo-tetrahedral) and the five-coordinate $\left[\mathrm{InCl}_{3}\left(\mathrm{AsEt}_{3}\right)_{2}\right]$ (trans-trigonal bipyramidal) are shown in Figure 3.

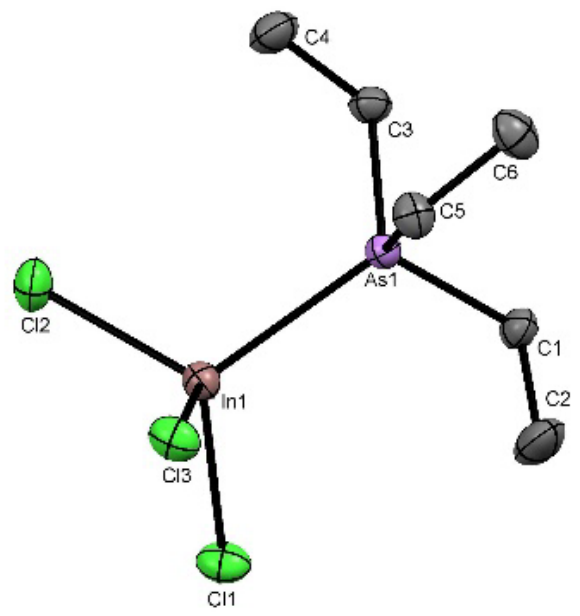

(a)

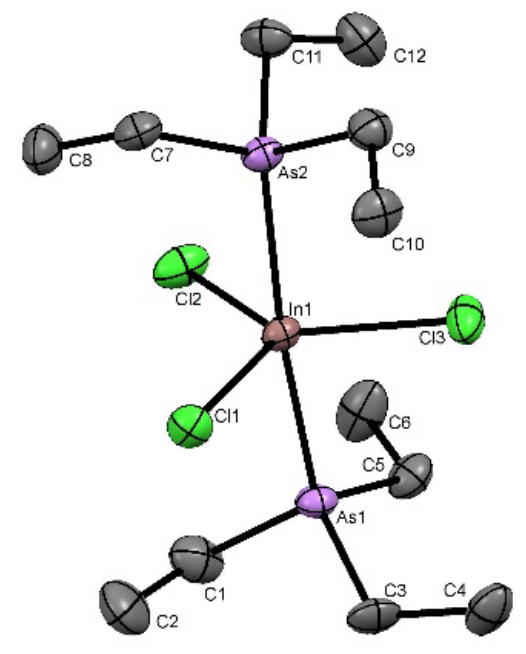

(b)

Figure 3. View of the structure of $\left[\operatorname{InCl}_{3}\left(\mathrm{AsEt}_{3}\right)\right]$ (a) with atom numbering scheme. $\mathrm{H}$ atoms are omitted for clarity and ellipsoids are shown at the $50 \%$ probability level. Selected bond lengths $(\AA)$ and angles $\left({ }^{\circ}\right): \ln 1-A s 1$ $=2.6055(4), \operatorname{In} 1-\mathrm{Cl} 3=2.3854(9), \operatorname{In} 1-\mathrm{Cl} 1=2.3761(9), \operatorname{In} 1-\mathrm{Cl} 2=2.3549(10), \mathrm{C} 1-\mathrm{As} 1-\mathrm{C} 5=106.63(16)$, 
C3-As1-C1 = 107.48(16), C3-As1-C5 = 106.34(16) and (b) $\left[\operatorname{InCl}_{3}\left(\mathrm{AsEt}_{3}\right)_{2}\right] \operatorname{In} 1-\mathrm{As} 2=2.7025(11), \operatorname{In} 1-\mathrm{As} 1=$ 2.6995(10), $\ln 1-\mathrm{Cl} 1=2.412(2), \ln 1-\mathrm{Cl} 2=2.431(2), \ln 1-\mathrm{Cl} 3=2.428(3), \mathrm{C} 9-\mathrm{As} 2-\mathrm{C} 7=103.1(5), \mathrm{C} 9-\mathrm{As} 211=$ 105.1(5), C11-As2-C7 = 103.2(5), C3-As1-C5 = 105.3(5), C1-As1-C3 = 102.4(5), C1-As1-C5 = 104.3(5), As1-In1-As2 = 172.98(4).

The In- $\mathrm{Cl}$ distances increase by $0.03-0.05 \AA$ A between the four- and five-coordinate complexes and the In-As similarly increases by $\sim 0.09 \AA$. The In-Cl distances are the same within experimental error in $\left[\operatorname{InCl}_{3}\left(\mathrm{AsEt}_{3}\right)\right]$ and $\left[\operatorname{InCl}_{3}\left(\mathrm{Sb}^{n} \mathrm{Bu}_{3}\right)\right]$, consistent with the $\mathrm{In}-\mathrm{Cl}$ being the dominant interaction. As demonstrated by DFT calculations [7], coordination of the pnictine ligand to the triel trihalide is accompanied by a widening of the $\mathrm{C}-\mathrm{Sb} / \mathrm{As}-\mathrm{C}$ angles due to changes in the $\mathrm{s}$ and $\mathrm{p}$ antimony/arsenic components of the C-Sb/As bonds compared to the free pnictine, the effect being greater in the stibine complexes.

\subsection{Low Pressure CVD Studies}

Low pressure CVD of metal chalcogenide films from the corresponding chalcogenoether complexes of the metal halides uses alkyl groups on the chalcogen with two or more carbon atoms, which makes the low energy $\beta$-hydride decomposition route available $[18,19]$. Successful depositions and the film quality often depend upon quite small changes in the reagents and conditions, and we have found n-butyl groups on the neutral ligand to be particularly favourable. In addition, the complexes of the $n$-butyl ligands are often oils or low melting solids, indicating rather low lattice energies, which may aid the vaporisation of the precursor on heating in vacuo. Hence, in the present study $\left[\operatorname{lnCl}_{3}\left(\mathrm{Sb}^{n} \mathrm{Bu}_{3}\right)\right]$ and $\left[\mathrm{GaCl}_{3}\left(\mathrm{Sb}^{n} \mathrm{Bu}_{3}\right)\right]$ were explored for low pressure CVD, as well as the known $\left[\operatorname{lnCl}_{3}\left(\mathrm{SbEt}_{3}\right)\right]$ and $\left[\mathrm{GaCl}_{3}\left(\mathrm{SbEt}_{3}\right)\right]$ [7]. Deposition of grey or black films on the tiles was observed in the majority of cases at temperatures $>500^{\circ} \mathrm{C}$ for the stibine complexes, but XRD measurements confirmed these to be hexagonal antimony with the space group R-3m, $\alpha=4.2967(3), b=4.2967(3)$, $c=11.2453(7) \AA$, in good agreement with the literature values $(a=4.3084(2), b=4.3084(2), c=$ 11.2740(2) ̊̊) [26]. In a few cases small island deposits shown to contain In and Cl were observed, but there was no evidence for InSb deposition. Attempts to produce InAs or GaAs from $\left[\operatorname{InCl}_{3}\left(\mathrm{AsEt}_{3}\right)\right]$ or $\left[\mathrm{GaCl}_{3}\left(\mathrm{AsEt}_{3}\right)\right]$ did not lead to any significant deposition.

\section{Conclusions}


A new series of triel trihalide stibine complexes $\left[\ln _{3}\left(\mathrm{Sb}^{n} \mathrm{Bu}_{3}\right)\right]$ and $\left[\mathrm{GaX}_{3}\left(\mathrm{Sb}^{n} \mathrm{Bu} u_{3}\right)\right](X=\mathrm{Cl}, \mathrm{Br}, \mathrm{I})$ has been prepared and fully characterised, including the X-ray crystal structures of $\left[\ln X_{3}\left(\mathrm{Sb}^{n} \mathrm{Bu}_{3}\right)\right](X=$ $\mathrm{Cl}, \mathrm{Br})$ and $\left[\mathrm{Gal}_{3}\left(\mathrm{Sb}^{\mathrm{n}} \mathrm{Bu}_{3}\right)\right]$. New complexes of $\mathrm{AsEt}_{3}$, specifically $\left[\mathrm{GaCl}_{3}\left(\mathrm{AsEt}_{3}\right)\right],\left[\mathrm{InCl}_{3}\left(\mathrm{AsEt}_{3}\right)\right]$ and $\left[\mathrm{InCl}_{3}\left(\mathrm{AsEt}_{3}\right)_{2}\right]$, the latter two being four- and five-coordinate, respectively, were also characterised crystallographically. Attempts to use $\left[\mathrm{InCl}_{3}\left(\mathrm{SbR}_{3}\right)\right]$ and $\left[\mathrm{GaCl}_{3}\left(\mathrm{SbR}_{3}\right)\right]\left(\mathrm{R}={ }^{\mathrm{n}} \mathrm{Bu}, \mathrm{Et}\right)$ as single source low pressure CVD reagents for InSb or GaSb thin films were unsuccesful, with only elemental antimony deposited, contrasting with the selenoether and telluroether analogues, $\left[\mathrm{GaCl} 3\left(\mathrm{E}^{\mathrm{n}} \mathrm{Bu}_{2}\right)\right](\mathrm{E}=\mathrm{Se}, \mathrm{Te})$, which produce thin films of the binary $\mathrm{Ga}_{2} \mathrm{Se}_{3}$ and $\mathrm{Ga}_{2} \mathrm{Te}_{3}$ materials under analogous conditions [16].

\section{Acknowledgements}

We thank EPSRC for support (EP/P025137/1) and for studentships to KRC (EP/R513325/1) and FR (EP/N509747/1).

\section{Conflicts of Interest}

The authors have no conflicts to declare.

\section{Appendix A}

The supplementary material for this paper including the X-ray data, IR and multinuclear NMR spectra of new complexes may be found at http:/

\section{References}

[1] N. R. Champness, W. Levason, Coord. Chem. Rev. 133 (1994) 115.

[2] W. Levason, G. Reid, Coord. Chem. Rev. 250 (2006) 2565.

[3] H. Werner, Angew. Chem. Int. Ed. 47 (2004) 938.

[4] S. L. Benjamin, T. Krämer, W. Levason, M. E. Light, S. A. Macgregor, G. Reid, J. Am. Chem. Soc. 138 (2016) 6964.

[5] S. L. Benjamin, G. Reid, Coord. Chem. Rev. 297-298 (2015) 168.

[6] J. Burt, W. Levason, G. Reid, Coord. Chem. Rev. 260 (2014) 65.

[7] V. K. Greenacre, W. Levason, G. Reid, Organometallics 37 (2018) 2123.

[8] S. Mokkapati, C. Jagadish, Mater. Today 12 (2009) 22. 
[9] P. Marchand, S. Sathasivam, B. A. D. Wilkinson, D. Pugh, S. M. Bawaked, S. N. Basahel, A. Y. Obaid, D. O. Scanlon, I. P. Parkin, C. J. Carmalt, J. Mater. Chem. C 4 (2016) 6761.

[10] K. E. Hnida, S. Bäßler, J. Mech, K. Szaciłowski, R. P. Socha, M. Gajewska, K. Nielsch, M. Przybylski, G. D. Sulka, J. Mater. Chem. C 4 (2016) 1345.

[11] A. K. Sharma, P. J. Reddy, J. Non-cryst. Solids 41 (1980) 13-30.

[12] M. Singh, Y. K. Vijay, Indian J. Pure \& App. Phys. 42 (2004) 610.

[13] J. H. Dias da Silva, J. I. Cisneros, M. M. Guraya, G. Zampieri, Phys. Rev. B 51 (1995) 6272.

[14] P. N. Bartlett, D. Cook, C. H. de Groot, A. L. Hector, R. Huang, A. Jolleys, G. P. Kissling, W. Levason, S. J. Pierce, G. Reid, RSC Advances 3 (2013) 15645.

[15] A. H. Cowley, R. A. Jones, C. M. Nunn, D. L. Westmorland, Chem. Mater. 2 (1990) 221.

[16] K. George, C. H. de Groot, C. Gurnani, A. L. Hector, R. Huang, M. Jura, W. Levason, G. Reid, Chem. Mater. 25 (2013) 1829.

[17] G. M. Sheldrick, Acta Crystallogr. Sect. C 71 (2015) 3.

[18] S. L. Benjamin, Y-P. Chang, C. Gurnani, A. L. Hector, M. Huggon, W. Levason, G. Reid, Dalton Trans. 43 (2014) 16640.

[19] S. L. Benjamin, C. H. de Groot, A. L. Hector, R. Huang, E. Koukharenko, W. Levason, G. Reid, J. Mat. Chem. C. $3(2015) 423$.

[20] A. J. Carty, T. Hinsperger, P. M. Boorman, Can. J. Chem. 48 (1970) 1959.

[21] M. V. Veidis, G. J. Palenik, Chem. Commun. (1969) 586.

[22] M. A. Brown, D. G. Tuck, E. J. Wells, Can. J. Chem. 74 (1996) 1535.

[23] L.-J. Baker, L. A. Kloo, C. E. F. Rickard, M. J. Taylor, J. Organomet. Chem. 545-546 (1997) 249.

[24] J. Emsley, The Elements, OUP Oxford 1989.

[25] F. Cheng, A. L. Hector, W. Levason, G. Reid, M. Webster, W. Zhang, Inorg. Chem. 46 (2007) 7251.

[26] C. S. Barrett, P. Cucka, K. Haefner, Acta Cryst. 16 (1963) 451. 